\title{
Ear Drops Solution Dosage Form
}

National Cancer Institute

\section{Source}

National Cancer Institute. Ear Drops Solution Dosage Form. NCI Thesaurus. Code C149453.

Liquid single-dose or multidose preparation consisting of an aqueous or oily solution intended for application to the external auditory meatus. Multidose containers may be dropper containers or containers provided with a dropper applicator, or the dropper may be supplied separately. Drops are not necessarily administered dropwise, but may also be administered as a small volume. 